J. Appl. Numer. Optim. 2 (2020), No. 3, pp. 335-351

Available online at http://jano.biemdas.com

https://doi.org/10.23952/jano.2.2020.3.05

\title{
AN INERTIAL MANN-LIKE ALGORITHM FOR FIXED POINTS OF NONEXPANSIVE MAPPINGS IN HILBERT SPACES
}

\author{
BING TAN ${ }^{1}$, SUN YOUNG CHO ${ }^{2, *}$ \\ ${ }^{1}$ Institute of Fundamental and Frontier Sciences, \\ University of Electronic Science and Technology of China, Chengdu, China \\ ${ }^{2}$ Department of Liberal Arts, Gyeongnam National University of Science and Technology, Jinju-Si, Korea
}

\begin{abstract}
In this paper, we investigate an inertial Mann-like algorithm for fixed points of nonexpansive mappings in Hilbert spaces and obtain strong convergence results under some mild assumptions. Based on this, we derive a forward-backward algorithm involving Tikhonov regularization terms, which converges strongly to the solution of the monotone inclusion problem. We demonstrate the advantages of our algorithms comparing with some existing ones in the literature via split feasibility problem, variational inequality problem and signal recovery problem.
\end{abstract}

Keywords. Inertial Mann algorithm; Forward-backward splitting algorithm; Tikhonov regularization; Nonexpansive mapping; Strong convergence.

\section{INTRODUCTION}

Let $C$ be a nonempty convex closed subset in a real Hilbert space $\mathscr{H}$, where $\mathscr{H}$ endowed with inner product $\langle\cdot, \cdot\rangle$ and induced norm $\|\cdot\|:=\sqrt{\langle\cdot, \cdot\rangle}$. For all $x, y \in C$, recall that a mapping $T$ : $C \rightarrow C$ is said to be (i) $L$-Lipschitzian iff $\|T x-T y\| \leq L\|x-y\|$ for some $L>0$; (ii) nonexpansive if $\|T x-T y\| \leq\|x-y\|$. We denote $\operatorname{Fix}(T):=\{x \in C: T x=x\}$ as the set of fixed points of mapping $T$. The main purpose of this paper is to design a fast Mann-like iterative algorithm to solve fixed point problems of nonexpansive mappings. It is well known that the Mann algorithm is one of the numerous successful iterative schemes for finding a fixed point of nonexpansive mappings. The Mann algorithm reads as follows:

$$
x_{n+1}=\left(1-\lambda_{n}\right) x_{n}+\lambda_{n} T x_{n}, \quad \forall n \geq 0,
$$

where $\left\{\lambda_{n}\right\}$ is a sequence of non-negative real numbers in $(0,1)$, and $x_{0} \in \mathscr{H}$ is an arbitrary initial point. Under the assumption that $\operatorname{Fix}(T) \neq \emptyset$, it is known that the sequence $\left\{x_{n}\right\}$ defined by (1.1) converges weakly to a fixed point of $T$ under suitable conditions forced on $\left\{\lambda_{n}\right\}$.

The fixed point problems of nonexpansive mappings involve many important issues, such as, machine learning, signal restoration, image reconstruction, and so on; see, e.g., [1, 15, 19] and the references therein. Therefore, devising efficient and stable algorithms has attracted tremendous interest. It is known that the forward-backward algorithm and the Douglas-Rachford

${ }^{*}$ Corresponding author.

E-mail addresses: bingtan72@gmail.com (B. Tan), sycho@gntech.ac.kr (S.Y. Cho).

Received March 3, 2020; Accepted November 4, 2020.

(C)2020 Journal of Applied and Numerical Optimization 
algorithm can be embedded in the framework of the Mann algorithm. One disadvantage to all the above algorithms is that their generated iterative sequences are only weakly convergent. However, for problems that occur in infinite-dimensional spaces, such as, image reconstruction and quantum physics, the weak convergence is often disappointing. To obtain strong convergence, it is usually necessary to require more restrictive properties on the operators involved, such as, strong convexity when considering optimization problems and strong monotonicity when analyzing monotone inclusion. Because there are situations for which these conditions are not satisfied, the development of efficient iterative algorithms is under the spotlight. In the past two decades, there has been extensive studies and applications of strong convergence algorithms; see, e.g., $[2,7,20]$ and the references therein. Very recently, a variant of the Mann algorithm was proposed by Bot, Csetneki and Meier [4], which overwhelms the shortcoming of the weak convergence and reads as follows:

$$
x_{n+1}=\beta_{n} x_{n}+\lambda_{n}\left(T\left(\beta_{n} x_{n}\right)-\beta_{n} x_{n}\right), \quad \forall n \geq 0 .
$$

They showed the iterative sequence $\left\{x_{n}\right\}$ defined by (1.2) is strongly convergent when $\left\{\lambda_{n}\right\}$ and $\left\{\beta_{n}\right\}$ satisfies some suitable assumptions. Based on (1.2), they obtained a strongly convergent forward-backward algorithm for monotone inclusion problems. We emphasize that $\left\{\beta_{n}\right\}$ in the above scheme is the Tikhonov regularization sequence, which plays the role to enforce the strong convergence property. More theoretical results and motivational arguments of the Tikhonov regularization technique can be found in [3]. For some numerical algorithms to implement the strong convergence by means of this technique, we recommend readers refer to [4, 22].

In recent years, various fast iterative algorithms have been investigated. One of the algorithms is the inertial algorithm, which can be traced back to Polyak [18]. Polyak first introduced an inertial extrapolation technique for minimizing a smooth convex function. One of the common characteristics of the inertial algorithms is that the next iterate depends on the combination of the previous two iterates. It should be mentioned that this minor change greatly improves the performance of the algorithms. With the help of inertial techniques, numerous fast iterative algorithms have been constructed, such as, inertial Mann algorithms [17, 28], inertial forward-backward splitting algorithms [21, 27], inertial projection algorithms [24, 26] and inertial extragradient algorithms $[10,12]$. These inertial-type algorithms have better numerical performance than algorithms without inertial terms.

Inspired and motivated by the above results, we propose an inertial Mann-like algorithm with the aid of the famous Nesterov acceleration method for fixed points of nonexpansive mappings in infinite-dimensional Hilbert spaces. We also derive a forward-backward algorithm endowed with Tikhonov regularization terms, which generate an iterative sequence that converges strongly to the minimal norm solution in the set of zeros. Finally, we provide some numerical experiments that demonstrate the advantages of our proposed algorithms by comparing them with some related ones. The algorithms obtained in this paper improve some known results in the literature.

The present paper is built up as follows. In the next section, we provide some preliminary knowledge and lemmas needed to perform our convergence analysis. Section 3 presents a strongly convergent inertial Mann-like algorithm and analyzes its convergence. Section 4 shows the applications of our algorithm to the problems of monotone inclusion and variational inequality. Section 5 illustrates the potential of our algorithms using numerical examples on split feasibility problems in infinite-dimensional Hilbert spaces, variational inequality problems, and signal recovery. Finally, it concludes this paper with a brief summary in Section 6, the last section. 


\section{PRELIMINARIES}

Throughout this paper, the notations $\rightarrow$ and $\rightarrow$ denote strong and weak convergence, respectively. Let $\mathscr{H}$ be a real Hilbert space. For any $x, y \in \mathscr{H}$, one has: (i) $\|x-y\|^{2}=$ $\|x\|^{2}+\|y\|^{2}-2\langle x, y\rangle$; (ii) $\|x+y\|^{2} \leq\|x\|^{2}+2\langle y, x+y\rangle$. Let $\alpha \in(0,1)$ be fixed. We recall that an operator $R: \mathscr{H} \rightarrow \mathscr{H}$ is said to be $\alpha$-averaged, if $R$ can be expressed as $R=(1-\alpha) \mathrm{Id}+\alpha T$, where $T: \mathscr{H} \rightarrow \mathscr{H}$ is a nonexpansive operator and Id is an identity operator defined on $\mathscr{H}$. It is easy to prove that $\alpha$-averaged operators are also nonexpansive. Let $A: \mathscr{H} \rightrightarrows \mathscr{H}$ be a set-valued operator, and its graph is represented as $\operatorname{Graph} A:=\{(x, p) \in \mathscr{H} \times \mathscr{H}: p \in A x\}$. The operator $A$ is said to be (i) monotone, if $\langle x-y, p-q\rangle \geq 0$ for all $(x, p),(y, q) \in \operatorname{Graph} A$; (ii) maximally monotone if it is monotone and there exists no proper monotone extension of the graph of $A$ on $\mathscr{H} \times \mathscr{H}$; (iii) $\eta$-inverse strongly monotone if there exists a constant $\eta>0$ such that $\langle x-y, A x-A y\rangle \geq \eta\|A x-A y\|^{2}$ for all $x, y \in \mathscr{H}$. The resolvent of $A, J_{A}: \mathscr{H} \rightarrow \mathscr{H}$, is defined by $J_{A}:=(\mathrm{Id}+A)^{-1}$. Further, we denote by $R_{A}: \mathscr{H} \rightarrow \mathscr{H}, R_{A}:=2 J_{A}-\mathrm{Id}$, the reflected resolvent of $A$. If $A$ is maximally monotone, then $J_{A}: \mathscr{H} \rightarrow \mathscr{H}$ is single-valued, maximally monotone and nonexpansive (see [6]). Moreover, $0 \in A(x)+B(x)$ if and only if $x=J_{\gamma A}(I-\gamma B)(x), \forall \gamma>0$. If $B$ is $\eta$-inverse strongly monotone with $0<\gamma<2 \eta$, then $J_{\gamma A}(I-\gamma B)$ is averaged.

We recall that if $f: \mathscr{H} \rightarrow \mathbb{R} \cup\{+\infty\}$ is a proper, convex and lower semicontinuous function, then the subdifferential of $f, \partial f: \mathscr{H} \rightrightarrows \mathscr{H}$, is defined by

$$
\partial f(x):=\{u \in \mathscr{H}: f(y)-f(x) \geq\langle u, y-x\rangle, \forall y \in \mathscr{H}\},
$$

for $x \in \mathscr{H}$ with $f(x) \neq+\infty$. We denote the proximal operator of $f$ as follows:

$$
\operatorname{prox}_{f}: \mathscr{H} \rightarrow \mathscr{H}, \operatorname{prox}_{f}(x):=\operatorname{argmin}_{y \in \mathscr{H}}\left\{\frac{1}{2}\|y-x\|^{2}+f(y)\right\} .
$$

It should be noted that the proximal operator of $f$ equivalent to the resolvent of $\partial f$ (see [6]), i.e., $\operatorname{prox}_{f}=J_{\partial f}$. For a nonempty closed and convex set $C \subseteq \mathscr{H}$, one sees that $P_{C}=\operatorname{prox}_{\delta_{C}}$, where

$$
\delta_{C}(x)= \begin{cases}0, & x \in C ; \\ +\infty, & x \notin C,\end{cases}
$$

denotes the indicator function of $C$. From the Baillon-Haddad Theorem (see [6, Corollary 18.16]), if $g: \mathscr{H} \rightarrow \mathbb{R}$ is a convex and Fréchet differentiable function with $\frac{1}{\eta}$-Lipschitz gradient, then $\nabla g: \mathscr{H} \rightarrow \mathscr{H}$ is an $\eta$-inverse strongly monotone operator.

In the rest of this section, we recall some lemmas that will play an indispensable role in the convergence analysis of the proposed algorithm.

Lemma 2.1 (Demi-closed principle). Assume that $C$ is a nonempty closed convex subset of a real Hilbert space $\mathscr{H}$. Let $T: C \rightarrow \mathscr{H}$ be a nonexpansive mapping. Let $\left\{x_{n}\right\}$ be a sequence in $C$ and $x \in \mathscr{H}$ such that $x_{n} \rightarrow x$ and $T x_{n}-x_{n} \rightarrow 0$ as $n \rightarrow+\infty$. Then $x \in \operatorname{Fix}(T)$.

Lemma 2.2. [29] Let $\left\{a_{n}\right\}$ be a sequence of non-negative real numbers such that

$$
a_{n+1} \leq\left(1-\mu_{n}\right) a_{n}+\tau_{n}+v_{n}, \quad n \geq 1,
$$

where $\left\{\mu_{n}\right\}$ is a sequence in $(0,1)$ and $\left\{\tau_{n}\right\}$ is a real sequence. Assume that $\sum_{n=1}^{\infty} v_{n}<\infty$. Then, the following results hold:

(i) If $\tau_{n} \leq \mu_{n} M$ for some $M \geq 0$, then $\left\{a_{n}\right\}$ is a bounded sequence;

(ii) If $\sum_{n=1}^{\infty} \mu_{n}=\infty$ and $\lim \sup _{n \rightarrow \infty} \frac{\tau_{n}}{\mu_{n}} \leq 0$, then $\lim _{n \rightarrow \infty} a_{n}=0$. 


\section{A STRONGLY CONVERGENT INERTIAL MANN-LIKE ALGORITHM}

Theorem 3.1. Let $C$ be a closed convex subset of a real Hilbert space $\mathscr{H}$ and let $T: C \rightarrow C$ be a nonexpansive mapping such that $\operatorname{Fix}(T) \neq \emptyset$. Suppose that the following conditions are satisfied:

(C1) $\lim _{n \rightarrow+\infty} \alpha_{n}=0, \sum_{n=0}^{\infty} \alpha_{n}=\infty, \alpha_{n} \in(0,1), \beta_{n} \in[a, b] \subset(0,1]$;

(C2) $\lim _{n \rightarrow+\infty} \frac{\theta_{n}}{\alpha_{n}}\left\|x_{n}-x_{n-1}\right\|=0$.

Set $x_{0}, x_{1}$ arbitrarily. Define a sequence $\left\{x_{n}\right\}$ in $C$ by the following iterative scheme:

$$
\left\{\begin{array}{l}
w_{n}=x_{n}+\theta_{n}\left(x_{n}-x_{n-1}\right), \\
y_{n}=\left(1-\alpha_{n}\right) w_{n}, \\
x_{n+1}=\left(1-\beta_{n}\right) y_{n}+\beta_{n} T y_{n}, n \geq 1 .
\end{array}\right.
$$

Then the iterative sequence $\left\{x_{n}\right\}$ defined by (3.1) converges to a fixed point of $T$ in norm.

Proof. To begin with, our first goal is to show that $\left\{x_{n}\right\}$ is bounded. Indeed, For any $u \in \operatorname{Fix}(T)$, since $T$ is nonexpansive, one sees that

$$
\begin{aligned}
\langle T x-u, T x-u\rangle & \leq\langle x-u, x-u\rangle \\
& \Longrightarrow\langle T x-u, T x-u\rangle \leq\langle x-u, x-T x\rangle+\langle x-u, T x-u\rangle \\
& \Longrightarrow\langle T x-u, T x-x\rangle \leq\langle x-u, x-T x\rangle \\
\Longrightarrow & \Longrightarrow T x-x, T x-x\rangle+\langle x-u, T x-x\rangle \leq\langle x-u, x-T x\rangle \\
& \Longrightarrow\|T x-x\|^{2} \leq 2\langle x-u, x-T x\rangle .
\end{aligned}
$$

From the definition of $x_{n+1}$ and (3.2), we obtain

$$
\begin{aligned}
\left\|x_{n+1}-u\right\|^{2} & =\left\|\left(y_{n}-u\right)-\beta_{n}\left(y_{n}-T y_{n}\right)\right\|^{2} \\
& =\left\|y_{n}-u\right\|^{2}-2 \beta_{n}\left\langle y_{n}-u, y_{n}-T y_{n}\right\rangle+\beta_{n}^{2}\left\|y_{n}-T y_{n}\right\|^{2} \\
& \leq\left\|y_{n}-u\right\|^{2}-\beta_{n}\left(1-\beta_{n}\right)\left\|y_{n}-T y_{n}\right\|^{2} \\
& \leq\left\|y_{n}-u\right\|^{2},
\end{aligned}
$$

which yields

$$
\begin{aligned}
\left\|x_{n+1}-u\right\| & \leq\left\|y_{n}-u\right\|=\left\|\left(1-\alpha_{n}\right)\left(w_{n}-u\right)-\alpha_{n} u\right\| \\
& \leq\left(1-\alpha_{n}\right)\left\|w_{n}-u\right\|+\alpha_{n}\|u\| \\
& \leq\left(1-\alpha_{n}\right)\left\|x_{n}-u\right\|+\alpha_{n}\|u\|+\left(1-\alpha_{n}\right) \theta_{n}\left\|x_{n}-x_{n-1}\right\| .
\end{aligned}
$$

Due to the fact that $\sup _{n \geq 1} \frac{\theta_{n}}{\alpha_{n}}\left\|x_{n}-x_{n-1}\right\|$ exists. Let

$$
M:=2 \max \left\{\|u\|, \sup _{n \geq 1} \frac{\left(1-\alpha_{n}\right) \theta_{n}}{\alpha_{n}}\left\|x_{n}-x_{n-1}\right\|\right\} .
$$

As a result, inequality (3.4) is reduced to the following

$$
\left\|x_{n+1}-u\right\| \leq\left(1-\alpha_{n}\right)\left\|x_{n}-u\right\|+\alpha_{n} M .
$$

Using Lemma 2.2, it is easy to check that $\left\{x_{n}\right\}$ is bounded. On the other hand, according to the definition of $w_{n}$, one has

$$
\left\|w_{n}-u\right\|^{2}=\left\|x_{n}-u\right\|^{2}+2 \theta_{n}\left\langle x_{n}-x_{n-1}, x_{n}-u\right\rangle+\theta_{n}^{2}\left\|x_{n}-x_{n-1}\right\|^{2} .
$$


Combining boundedness of $\left\{x_{n}\right\}$ and condition (C2), we observe that $\left\{w_{n}\right\}$ is bounded, and thus $\left\{y_{n}\right\}$ is also bounded. From (3.1), we note that

$$
y_{n}-T y_{n}=1 / \beta_{n} \cdot\left(y_{n}-x_{n+1}\right),
$$

and

$$
\left\|w_{n}-x_{n+1}\right\|^{2}=\left\|x_{n}-x_{n+1}\right\|^{2}+2 \theta_{n}\left\langle x_{n}-x_{n+1}, x_{n}-x_{n-1}\right\rangle+\theta_{n}^{2}\left\|x_{n}-x_{n-1}\right\|^{2} .
$$

By using (3.3) and (3.6), we obtain

$$
\begin{aligned}
\left\|x_{n+1}-u\right\|^{2} & \leq\left\|y_{n}-u\right\|^{2}-\beta_{n}\left(1-\beta_{n}\right)\left\|y_{n}-T y_{n}\right\|^{2} \\
& \leq\left\|y_{n}-u\right\|^{2}-\frac{1-\beta_{n}}{\beta_{n}}\left\|y_{n}-x_{n+1}\right\|^{2} .
\end{aligned}
$$

Since $0<a<\beta_{n} \leq b \leq 1$ and $\frac{1-\beta_{n}}{\beta_{n}} \geq \frac{1-b}{b}=k$, from (3.5), (3.7) and (3.8), we have

$$
\begin{aligned}
\left\|x_{n+1}-u\right\|^{2} \leq & \left\|y_{n}-u\right\|^{2}-k\left\|y_{n}-x_{n+1}\right\|^{2} \\
= & \left\|w_{n}-u-\alpha_{n} w_{n}\right\|^{2}-k\left\|w_{n}-x_{n+1}-\alpha_{n} w_{n}\right\|^{2} \\
= & \left\|w_{n}-u\right\|^{2}-2 \alpha_{n}\left\langle w_{n}, w_{n}-u\right\rangle+\alpha_{n}^{2}\left\|w_{n}\right\|^{2} \\
& -k\left\|w_{n}-x_{n+1}\right\|^{2}+2 k \alpha_{n}\left\langle w_{n}, w_{n}-x_{n+1}\right\rangle-k \alpha_{n}^{2}\left\|w_{n}\right\|^{2} \\
= & \left\|w_{n}-u\right\|^{2}-k\left\|x_{n+1}-w_{n}\right\|^{2}+\alpha_{n}\left\{-2\left\langle w_{n}, w_{n}-u\right\rangle\right. \\
& \left.+2 k\left\langle w_{n}, w_{n}-x_{n+1}\right\rangle+(1-k) \alpha_{n}\left\|w_{n}\right\|^{2}\right\} \\
= & \left\|x_{n}-u\right\|^{2}-k\left\|x_{n}-x_{n+1}\right\|^{2}+2 \theta_{n}\left\langle x_{n}-x_{n-1}, x_{n}-u\right\rangle \\
& +2 k \theta_{n}\left\langle x_{n}-x_{n+1}, x_{n}-x_{n-1}\right\rangle+(1-k) \theta_{n}^{2}\left\|x_{n}-x_{n-1}\right\|^{2} \\
& +\alpha_{n}\left\{-2\left\langle w_{n}, w_{n}-u\right\rangle+2 k\left\langle w_{n}, w_{n}-x_{n+1}\right\rangle+(1-k) \alpha_{n}\left\|w_{n}\right\|^{2}\right\} .
\end{aligned}
$$

Because $\left\{x_{n}\right\}$ and $\left\{w_{n}\right\}$ are bounded, we have that there exists a constant $M_{1} \geq 0$ such that

$$
-2\left\langle w_{n}, w_{n}-u\right\rangle+2 k\left\langle w_{n}, w_{n}-x_{n+1}\right\rangle+(1-k) \alpha_{n}\left\|w_{n}\right\|^{2} \leq M_{1}, \forall n \geq 0 .
$$

Let

$$
M_{2}=2 \theta_{n}\left\langle x_{n}-x_{n-1}, x_{n}-u\right\rangle+2 k \theta_{n}\left\langle x_{n}-x_{n+1}, x_{n}-x_{n-1}\right\rangle+(1-k) \theta_{n}^{2}\left\|x_{n}-x_{n-1}\right\|^{2} .
$$

It follows from (3.9) that

$$
\left\|x_{n+1}-u\right\|^{2}-\left\|x_{n}-u\right\|^{2}+k\left\|x_{n+1}-x_{n}\right\|^{2} \leq \alpha_{n} M_{1}+M_{2} \text {. }
$$

Next we prove that $\left\{x_{n}\right\}$ converges strongly to $u$ by considering two possible cases.

Case 1. Suppose that the sequence $\left\{\left\|x_{n}-u\right\|\right\}$ is a monotonically decreasing sequence. Then $\left\{\left\|x_{n}-u\right\|\right\}$ is convergent. Obviously, we obtain $\left\|x_{n+1}-u\right\|^{2}-\left\|x_{n}-u\right\|^{2} \rightarrow 0$, which together with conditions (C1), (C2) and (3.10) indicates that

$$
\left\|x_{n+1}-x_{n}\right\| \rightarrow 0
$$

On the other hand, it is easy to see that

$$
\left\|w_{n}-x_{n+1}\right\| \leq\left\|x_{n}-x_{n+1}\right\|+\theta_{n}\left\|x_{n}-x_{n-1}\right\| \rightarrow 0,
$$

and

$$
\left\|y_{n}-w_{n}\right\| \leq \alpha_{n}\left\|w_{n}\right\| \rightarrow 0
$$


It follows from (3.12) and (3.13) that

$$
\left\|y_{n}-x_{n+1}\right\| \leq\left\|y_{n}-w_{n}\right\|+\left\|w_{n}-x_{n+1}\right\| \rightarrow 0 .
$$

By using (3.11) and (3.14), we have

$$
\left\|y_{n}-x_{n}\right\| \leq\left\|y_{n}-x_{n+1}\right\|+\left\|x_{n+1}-x_{n}\right\| \rightarrow 0,
$$

and

$$
\left\|y_{n}-T y_{n}\right\| \leq 1 / \beta_{n} \cdot\left\|y_{n}-x_{n+1}\right\| \rightarrow 0 .
$$

Combining (3.15) and (3.16), one sees that

$$
\begin{aligned}
\left\|x_{n}-T x_{n}\right\| & \leq\left\|x_{n}-y_{n}\right\|+\left\|y_{n}-T y_{n}\right\|+\left\|T y_{n}-T x_{n}\right\| \\
& \leq 2\left\|x_{n}-y_{n}\right\|+\left\|y_{n}-T y_{n}\right\| \rightarrow 0 .
\end{aligned}
$$

Therefore, from the fact that $I-T$ is demi-closed, we can easily proved that $\left\{x_{n}\right\}$ and $\left\{y_{n}\right\}$ converge weakly to a fixed point of $T$.

Next, it remains to show that $\left\{x_{n}\right\}$ and $\left\{y_{n}\right\}$ converge strongly to $u$. In view of (3.4), we get

$$
\begin{aligned}
\left\|x_{n+1}-u\right\|^{2} \leq & \left\|y_{n}-u\right\|^{2}=\left\|\left(1-\alpha_{n}\right)\left(w_{n}-u\right)-\alpha_{n} u\right\|^{2} \\
\leq & \left(1-\alpha_{n}\right)\left\|w_{n}-u\right\|^{2}-2 \alpha_{n}\left\langle y_{n}-u, u\right\rangle \\
\leq & \left(1-\alpha_{n}\right)\left\|x_{n}-u\right\|^{2}+2 \theta_{n}\left\langle x_{n}-x_{n-1}, x_{n}-u\right\rangle \\
& +\theta_{n}^{2}\left\|x_{n}-x_{n-1}\right\|^{2}-2 \alpha_{n}\left\langle y_{n}-u, u\right\rangle .
\end{aligned}
$$

Due to conditions $(\mathrm{C} 1)$ and $(\mathrm{C} 2)$, we deduce that

$$
2 \theta_{n}\left\langle x_{n}-x_{n-1}, x_{n}-u\right\rangle+\theta_{n}^{2}\left\|x_{n}-x_{n-1}\right\|^{2}-2 \alpha_{n}\left\langle y_{n}-u, u\right\rangle \rightarrow 0 .
$$

Thus, we get from Lemma 2.2 and (3.18) that $x_{n} \rightarrow u$. Further, it is easy to verify that $y_{n} \rightarrow u$.

Case 2. Suppose that $\left\{\left\|x_{n}-u\right\|\right\}$ is not a monotonically decreasing sequence. Set $\Phi_{n}=$ $\left\|x_{n}-u\right\|^{2}$. Let $\tau(n): N \rightarrow N$ be a mapping for all $n \geq n_{0}$ (for some large enough $n_{0}$ ), defined by $\tau(n)=\max \left\{k \in N: k \leq n, \Phi_{k} \leq \Phi_{k+1}\right\}$. Obviously, $\tau(n)$ is non-decreasing with $\tau(n) \rightarrow \infty$ as $n \rightarrow \infty$ and $\Phi_{\tau(n)} \leq \Phi_{\tau(n)+1}$ for $n \geq n_{0}$. It follows from (3.10) that

$$
\left\|x_{\tau(n)+1}-x_{\tau(n)}\right\|^{2} \leq \frac{\alpha_{\tau(n)} M_{1}+M_{2}}{k} \rightarrow 0
$$

which implies that $\left\|x_{\tau(n)+1}-x_{\tau(n)}\right\| \rightarrow 0$ as $n \rightarrow \infty$. By the same argument as (3.12)-(3.17) in Case 1 , we infer directly that $x_{\tau(n)}$ and $y_{\tau(n)}$ converge weakly to $u$ as $\tau(n) \rightarrow \infty$. In addition, from (3.18), we note that, for all $n \geq n_{0}$,

$$
\begin{aligned}
0 \leq & \left\|x_{\tau(n)+1}-u\right\|^{2}-\left\|x_{\tau(n)}-u\right\|^{2} \\
\leq & \alpha_{\tau(n)}\left[2\left\langle u-y_{\tau(n)}, u\right\rangle-\left\|x_{\tau(n)}-u\right\|^{2}\right] \\
& +2 \theta_{\tau(n)}\left\langle x_{\tau(n)}-x_{\tau(n)-1}, x_{\tau(n)}-u\right\rangle+\theta_{\tau(n)}^{2}\left\|x_{\tau(n)}-x_{\tau(n)-1}\right\|^{2},
\end{aligned}
$$

which implies that

$$
\begin{aligned}
\left\|x_{\tau(n)}-u\right\|^{2} \leq & 2\left\langle u-y_{\tau(n)}, u\right\rangle+\frac{2 \theta_{\tau(n)}\left\langle x_{\tau(n)}-x_{\tau(n)-1}, x_{\tau(n)}-u\right\rangle}{\alpha_{\tau(n)}} \\
& +\frac{\theta_{\tau(n)}^{2}\left\|x_{\tau(n)}-x_{\tau(n)-1}\right\|^{2}}{\alpha_{\tau(n)}} .
\end{aligned}
$$


Hence, combining (3.19) and condition (C2), we deduce that $\lim _{n \rightarrow \infty}\left\|x_{\tau(n)}-u\right\|=0$. Thus, $\lim _{n \rightarrow \infty} \Phi_{\tau(n)}=\lim _{n \rightarrow \infty} \Phi_{\tau(n)+1}=0$. Moreover, for any $n \geq n_{0}$, it is easy to see that $\Phi_{n} \leq \Phi_{\tau(n)+1}$ if $n \neq \tau(n)$ (i.e., $\tau(n)<n$ ), since $\Phi_{j}>\Phi_{j+1}$ for $\tau(n)+1 \leq j \leq n$. Consequently, for all $n \geq n_{0}$,

$$
0 \leq \Phi_{n} \leq \max \left\{\Phi_{\tau(n)}, \Phi_{\tau(n)+1}\right\}=\Phi_{\tau(n)+1} .
$$

Therefore, we conclude that $\lim _{n \rightarrow \infty} \Phi_{n}=0$. This shows that sequence $\left\{x_{n}\right\}$ converges to $u$ in norm. Moreover, it easy to check that $\left\{y_{n}\right\}$ converges to $u$ in norm. The proof is completed.

Remark 3.1. From Algorithm (3.1), we have the following observations:

(i) We comment here that condition (C2) is easy to implement in numerical computation because the value of $\left\|x_{n}-x_{n-1}\right\|$ is known before choosing $\theta_{n}$. For a special choice, the parameter $\theta_{n}$ in the Algorithm (3.1) can be choosen from the following:

$$
0 \leq \theta_{n} \leq \bar{\theta}_{n}, \quad \bar{\theta}_{n}= \begin{cases}\min \left\{\frac{\xi_{n}}{\left\|x_{n}-x_{n-1}\right\|}, \frac{n-1}{n+\eta-1}\right\}, & \text { if } x_{n} \neq x_{n-1} \\ \frac{n-1}{n+\eta-1}, & \text { otherwise }\end{cases}
$$

for some $\eta \geq 3$ and $\left\{\xi_{n}\right\}$ is a positive sequence such that $\lim _{n \rightarrow \infty} \frac{\xi_{n}}{\alpha_{n}}=0$. This idea comes from the recent inertial extrapolated step introduced in [5].

(ii) For the Tikhonov regularization sequence $\left\{\alpha_{n}\right\}$, one can choose, for instance, $\alpha_{n}=\frac{1}{n+1}$ for any $n \geq 1$.

(iii) If $\theta_{n}=0$ for all $n \geq 1$ in Algorithm (3.1), then we obtain the results recently proposed by Bot, Csetnek and Meier [4]. It should be noted that our conditions are weaker than [4].

(iv) If $\alpha_{n}=0$ in Algorithm (3.1), then we get the inertial Mann algorithm proposed by Maingé [17].

In the next section, the following result will play a decisive role in the converge analysis of the forward-backward method endowed with the Tikhonov regularization term.

Corollary 3.1. Let $C$ be a closed convex subset of a real Hilbert space $\mathscr{H}$ and let $R: C \rightarrow C$ be an $\alpha$-averaged mapping, where $\alpha \in(0,1)$, with $\operatorname{Fix}(R) \neq \emptyset$. Assume that the following conditions are satisfied:

(D1) $\lim _{n \rightarrow+\infty} \alpha_{n}=0, \sum_{n=0}^{\infty} \alpha_{n}=\infty, \alpha_{n} \in(0,1), \beta_{n} \in[a, b] \subset\left(0, \frac{1}{\alpha}\right]$;

(D2) $\lim _{n \rightarrow+\infty} \frac{\theta_{n}}{\alpha_{n}}\left\|x_{n}-x_{n-1}\right\|=0$.

Set $x_{0}, x_{1}$ arbitrarily. Generate a sequence $\left\{x_{n}\right\}$ in $C$ by the following iterative scheme:

$$
\left\{\begin{array}{l}
w_{n}=x_{n}+\theta_{n}\left(x_{n}-x_{n-1}\right), \\
y_{n}=\left(1-\alpha_{n}\right) w_{n}, \\
x_{n+1}=\left(1-\beta_{n}\right) y_{n}+\beta_{n} R y_{n}, n \geq 1 .
\end{array}\right.
$$

Then the iterative sequence $\left\{x_{n}\right\}$ generated by (3.21) converges to a fixed point of $R$ in norm.

Proof. There exists a nonexpansive operator $T: \mathscr{H} \rightarrow \mathscr{H}$ such that $R=(1-\alpha) \mathrm{Id}+\alpha T$ since $R$ is $\alpha$-averaged operator. Observe that (3.21) is equivalent to

$$
\left\{\begin{array}{l}
w_{n}=x_{n}+\theta_{n}\left(x_{n}-x_{n-1}\right), \\
y_{n}=\left(1-\alpha_{n}\right) w_{n}, \\
x_{n+1}=\left(1-\alpha \beta_{n}\right) y_{n}+\alpha \beta_{n} T y_{n}, n \geq 1,
\end{array}\right.
$$

and that $\operatorname{Fix}(R)=\operatorname{Fix}(T)$. From Theorem 3.1, we obtain the desired conclusion immediately. 


\section{ApPLiCATIONS}

4.1. Monotone inclusions. Based on the general iterative scheme in Theorem 3.1, we will develop a strongly convergent inertial forward-backward algorithm with Tikhonov regularization terms in this subsection to solve the following monotone inclusion problem

$$
\text { find } x \in \mathscr{H} \text { such that } 0 \in A x+B x \text {, }
$$

where $A: \mathscr{H} \rightrightarrows \mathscr{H}$ is a maximally monotone operator and $B: \mathscr{H} \rightarrow \mathscr{H}$ is a $\eta$-inverse strongly monotone operator. Monotone inclusion problem (4.1) include some practical problems in image processing, machine learning and linear inverse problem.

Theorem 4.1. Let $A: \mathscr{H} \rightrightarrows \mathscr{H}$ be a maximally monotone operator and let $B: \mathscr{H} \rightarrow \mathscr{H}$ be an $\eta$-inverse strongly monotone operator such that $\operatorname{Fix}(A+B) \neq \emptyset$. Let $\gamma \in(0,2 \eta]$. Assume that the following conditions are satisfied:

(M1) $\lim _{n \rightarrow+\infty} \alpha_{n}=0, \sum_{n=0}^{\infty} \alpha_{n}=\infty, \alpha_{n} \in(0,1), \beta_{n} \in[a, b] \subset(0,(4 \eta-\gamma) / 2 \eta]$;

(M2) $\lim _{n \rightarrow+\infty} \frac{\theta_{n}}{\alpha_{n}}\left\|x_{n}-x_{n-1}\right\|=0$.

Set $x_{0}, x_{1}$ arbitrarily. Make a sequence $\left\{x_{n}\right\}$ in $C$ by the following iterative scheme:

$$
\left\{\begin{array}{l}
w_{n}=x_{n}+\theta_{n}\left(x_{n}-x_{n-1}\right), \\
y_{n}=\left(1-\alpha_{n}\right) w_{n}, \\
x_{n+1}=\left(1-\beta_{n}\right) y_{n}+\beta_{n} J_{\gamma A}\left(y_{n}-\gamma B\left(y_{n}\right)\right), n \geq 1 .
\end{array}\right.
$$

Then the iterative sequence $\left\{x_{n}\right\}$ maked by (4.2) converges to $P_{(A+B)^{-1}}(0)$ in norm.

Proof. It is easy to see that iterative scheme (4.2) can be written as (3.1), where $T=J_{\gamma A} \circ$ $(\mathrm{Id}-\gamma B)$. We consider two situations to analyze it. The first one is $\gamma \in(0,2 \eta)$. By means of [6, Corollary 23.8 and Remark 4.24(iii)], $J_{\gamma A}$ is $1 / 2$-inverse strongly monotone. Furthermore, according to [6, Proposition 4.33], $\mathrm{Id}-\gamma B$ is $\gamma / 2 \eta$-averaged. Therefore, it follows from [4, Proposition 6] that $T$ is $2 \eta /(4 \eta-\gamma)$-averaged. The statement now comes from Corollary 3.1 , by noticing that $\operatorname{Fix}(T)=(A+B)^{-1}(0)$. The second case is $\gamma=2 \eta$. Note that $\mathrm{Id}-\gamma B$ is nonexpansive, and thus $T=J_{\gamma A} \circ(\mathrm{Id}-\gamma B)$ is also nonexpansive. From Theorem 3.1, we conclude the desired conclusion immediately.

Remark 4.1. From Theorem 4.1, we have the following conclusions:

(i) If $\beta_{n}=1$ and $B=0$, then iterative scheme (4.2) becomes $x_{n+1}=J_{\gamma A}\left(\left(1-\alpha_{n}\right) w_{n}\right)$. This equation can be equivalently written as

$$
w_{n} \in \frac{1}{1-\alpha_{n}} x_{n+1}+\frac{\gamma}{1-\alpha_{n}} A x_{n+1}=\left(\operatorname{Id}+\varepsilon_{n} \operatorname{Id}+\frac{\gamma}{1-\alpha_{n}} A\right)\left(x_{n+1}\right),
$$

where $\varepsilon_{n}$ Id (with $\varepsilon_{n}:=\frac{1}{1-\alpha_{n}}-1>0$ and $\lim _{n \rightarrow+\infty} \varepsilon_{n}=0$ ) denotes the Tikhonov regularization term, which enforces the strong convergence of iterative sequence $\left\{x_{n}\right\}$ to the minimal norm solution. For Tikhonov-like methods to solve monotone inclusion problems, we recommend the readers to $[4,16,23]$ and the references therein.

(ii) Let $f: \mathscr{H} \rightarrow(-\infty,+\infty)$ be a proper, convex and lower-semicontinuous function, and let $g: \mathscr{H} \rightarrow \mathbb{R}$ be a convex and Fréchet differentiable function with $\frac{1}{\eta}$-Lipschitz continuous 
gradient such that $\operatorname{argmin}(f+g) \neq \emptyset$. Let $\gamma \in(0,2 \eta]$. The following iteration scheme

$$
\left\{\begin{array}{l}
w_{n}=x_{n}+\theta_{n}\left(x_{n}-x_{n-1}\right), \\
y_{n}=\left(1-\alpha_{n}\right) w_{n}, \\
x_{n+1}=\left(1-\beta_{n}\right) y_{n}+\beta_{n} \operatorname{prox}_{\gamma f}\left(y_{n}-\gamma \nabla_{g}\left(y_{n}\right)\right), n \geq 1,
\end{array}\right.
$$

where initial points $x_{0}, x_{1} \in \mathscr{H}$ and $\left\{\theta_{n}\right\},\left\{\alpha_{n}\right\}$ and $\left\{\beta_{n}\right\}$ are real sequences satisfying the conditions (M1) and (M2) in Theorem 4.1. Then the sequence $\left\{x_{n}\right\}$ created by (4.3) converges to $P_{\operatorname{argmin}(f+g)}(0)$ in norm.

4.2. Variational inequalities. We consider the following variational inequality problem:

$$
\text { find } x^{*} \in C \text { such that }\left\langle A x^{*}, y-x^{*}\right\rangle \geq 0, \quad \forall y \in C,
$$

where $A: \mathscr{H} \rightarrow \mathscr{H}$ is an operator and $C$ is a nonempty convex closed subset of $\mathscr{H}$. It is known that $x^{*}$ is a solution of (4.4) iff $x^{*}=P_{C}\left(x^{*}-\lambda A x^{*}\right)$, where $\lambda$ is an arbitrary positive constant.

Theorem 4.2. Let $A: \mathscr{H} \rightarrow \mathscr{H}$ be a monotone and L-Lipschitz continuous operator on a nonempty closed and convex subset $C$ and $\lambda \in(0,1 / L)$. Set $T:=P_{C}(\mathrm{Id}-\lambda A)$. Assume that the following conditions are satisfied:

(V1) $\lim _{n \rightarrow+\infty} \alpha_{n}=0, \sum_{n=0}^{\infty} \alpha_{n}=\infty, \alpha_{n} \in(0,1), \beta_{n} \in[a, b] \subset(0,1]$;

(V2) $\lim _{n \rightarrow+\infty} \frac{\theta_{n}}{\alpha_{n}}\left\|x_{n}-x_{n-1}\right\|=0$.

Set $x_{0}, x_{1}$ arbitrarily. Define a sequence $\left\{x_{n}\right\}$ in $C$ by the following iterative scheme:

$$
\left\{\begin{array}{l}
w_{n}=x_{n}+\theta_{n}\left(x_{n}-x_{n-1}\right) \\
y_{n}=\left(1-\alpha_{n}\right) w_{n}, \\
x_{n+1}=\left(1-\beta_{n}\right) y_{n}+\beta_{n} P_{C}(\operatorname{Id}-\lambda A) y_{n}, n \geq 1 .
\end{array}\right.
$$

Then the iterative sequence $\left\{x_{n}\right\}$ defined by (4.5) converges to $P_{\mathrm{Fix}(T)}(0)$ in norm.

Proof. We can easily conclude from Theorem 3.1 since $T=P_{C}(\mathrm{Id}-\lambda A)$ is nonexpansive.

\section{NUMERICAL EXPERIMENTS}

In this section, we provide some numerical experiments that appear in finite and infinite dimensional spaces to demonstrate the computational performance of our proposed algorithms and compare them with some previously known ones. All the programs were implemented in MATLAB 2018a on a Intel(R) Core(TM) i5-8250U CPU @ 1.60GHz computer.

Example 5.1. Assume that $\mathscr{H}_{1}$ and $\mathscr{H}_{2}$ are real Hilbert spaces and $T: \mathscr{H}_{1} \rightarrow \mathscr{H}_{2}$ is a bounded linear operator. Let $C$ and $Q$ be nonempty closed and convex subsets of $\mathscr{H}_{1}$ and $\mathscr{H}_{2}$, respectively. For the first numerical experiment, we consider the split feasibility problem (SFP) in infinitedimensional Hilbert spaces, which reads as

$$
\text { find } x^{*} \in C \text { such that } T x^{*} \in Q \text {. }
$$

We will use the strongly convergent algorithm described in (4.3) to solve the (SFP). For this purpose, it should be mentioned that problem (5.1) can be equivalently expressed as

$$
\min _{x \in \mathscr{H}}\left\{\frac{1}{2}\left\|T x-P_{Q}(T x)\right\|^{2}+\delta_{C}(x)\right\} .
$$


We choose $f(x)=\delta_{C}(x)$ and $g(x)=\frac{1}{2}\left\|T x-P_{Q}(T x)\right\|^{2}$ under the framework of Remark 4.1 (ii). Note that $g$ is Fréchet differentiable with gradient $\nabla g=T^{*} \circ\left(\mathrm{Id}-P_{Q}\right) \circ T$, and it holds

$$
\left\|\nabla g\left(x_{1}\right)-\nabla g\left(x_{2}\right)\right\| \leq\|L\|^{2}\left\|x_{1}-x_{2}\right\|, \quad \forall x_{1}, x_{2} \in \mathscr{H} .
$$

Therefore, the gradient of $g$ is $\|L\|^{2}$-Lipschitz continuous. The iterative scheme stated in (4.3) applied to problem (5.1) reads:

$$
\left\{\begin{array}{l}
w_{n}=x_{n}+\theta_{n}\left(x_{n}-x_{n-1}\right), \\
y_{n}=\left(1-\alpha_{n}\right) w_{n}, \\
v_{n}=\left(T^{*} \circ\left(\operatorname{Id}-P_{Q}\right) \circ T\right) y_{n}, \\
x_{n+1}=\left(1-\beta_{n}\right) y_{n}+\beta_{n} P_{C}\left(y_{n}-\gamma v_{n}\right), n \geq 1,
\end{array}\right.
$$

where $0<\gamma<2 L$ with Lipschitz constant $L=1 /\|T\|^{2}$.

Let $L^{2}([0,2 \pi]):=\left\{f:[0,2 \pi] \rightarrow \mathbb{R}: \int_{0}^{2 \pi}|f(t)|^{2} \mathrm{~d} t<+\infty\right\}$. For any $f, g \in L^{2}([0,2 \pi])$, we consider $\mathscr{H}_{1}=\mathscr{H}_{2}=L^{2}([0,2 \pi])$ embedded with the inner product $\langle f, g\rangle:=\int_{0}^{2 \pi} f(t) g(t) \mathrm{d} t$ and the induced norm $\|f\|:=\left(\int_{0}^{2 \pi}|f(t)|^{2} \mathrm{~d} t\right)^{\frac{1}{2}}$. Consider the following nonempty closed and convex subsets $C$ and $Q$ in $L^{2}([0,2 \pi])$ :

$$
\begin{aligned}
& C=\left\{x \in L^{2}([0,2 \pi]) \mid \int_{0}^{2 \pi} x(t) \mathrm{d} t \leq 1\right\}, \\
& Q=\left\{x \in L^{2}([0,2 \pi])\left|\int_{0}^{2 \pi}\right| x(t)-\left.\sin (t)\right|^{2} \mathrm{~d} t \leq 16\right\} .
\end{aligned}
$$

Let $T: L^{2}([0,2 \pi]) \rightarrow L^{2}([0,2 \pi])$ be a bounded linear operator with its adjoint $T^{*}$. We define $(T x)(t):=x(t)$. As a result, we have $\left(T^{*} x\right)(t)=x(t)$ and $\|T\|=1$. It is clear that the solution set of (5.1) is nonempty since $x(t)=0$ is a solution.

Inspired by reference [6], we use the following formulas to calculate the projections onto the sets $C$ and $Q$, respectively.

$$
P_{C}(x)=\left\{\begin{array}{ll}
\frac{1-a}{4 \pi^{2}}+x, & a>1 ; \\
x, & a \leq 1 .
\end{array} \text { and } P_{Q}(x)= \begin{cases}\sin (\cdot)+\frac{4(x-\sin (\cdot))}{\sqrt{b}}, & b>16 ; \\
x, & b \leq 16,\end{cases}\right.
$$

where $a=\int_{0}^{2 \pi} x(t) \mathrm{d} t$ and $b=\int_{0}^{2 \pi}|x(t)-\sin (t)|^{2} \mathrm{~d} t$.

We compare the number of iterations of the variants without and with Tikhonov regularization terms of the Algorithm (5.2), for different starting values and different inertial terms (cf., Table 1). We use the following stopping criterion

$$
E_{n}=\frac{1}{2}\left\|P_{C}\left(x_{n}\right)-x_{n}\right\|^{2}+\frac{1}{2}\left\|P_{Q}\left(T x_{n}\right)-T x_{n}\right\|^{2}<10^{-3} .
$$

In (5.2), inertial term $\theta_{n}$ is updated by (3.20) with $\xi_{n}=\frac{10}{(n+1)^{2}}$ and $\eta=4$, take relaxation variables $\beta_{n}=0.9$, step sizes $\gamma=0.25$ for every $n \geq 1$ and Tikhonov regularization parameters $\alpha_{n}=\frac{1}{n+1}$ for every $n \geq 1$, respectively, $\alpha_{n}=0$ for every $n \geq 1$ for the variants without Tikhonov regularization terms. We use symbolic computation in MATLAB to implement these algorithms for generating the iterative sequence. The numerical results are shown in Table 1.

Remark 5.1. (i) As indicated in Table 1, the numerical experiments show that the proposed Algorithm (5.2) involving Tikhonov regularization terms outperforms the ones without 
TABLE 1. Comparison of the variants without and with Tikhonov regularization terms of Algorithm (5.2) in Example 5.1.

\begin{tabular}{ccccccc}
\hline & & \multicolumn{2}{c}{$\theta_{n} \neq 0$} & & \multicolumn{2}{c}{$\theta_{n}=0$} \\
\cline { 3 - 4 } \cline { 6 - 7 }$x_{0}$ & $x_{1}$ & $\alpha_{n}=0$ & $\alpha_{n}=\frac{1}{n+1}$ & & $\alpha_{n}=0$ & $\alpha_{n}=\frac{1}{n+1}$ \\
\hline$\frac{t^{2}}{10}$ & $\frac{t^{2}}{10}$ & 7 & 3 & & 14 & 3 \\
$\frac{t^{2}}{10}$ & $\frac{2^{t}}{2}$ & 18 & 6 & & 24 & 8 \\
$\frac{t^{2}}{10}$ & $\frac{\mathrm{e}^{t}}{2}$ & 29 & 10 & & 32 & 11 \\
$\frac{t^{2}}{10}$ & $\frac{\mathrm{e}^{t}}{2}+\frac{t^{2}}{24}$ & 33 & 13 & & 35 & 14 \\
\hline
\end{tabular}

Tikhonov regularization terms (i.e., $\alpha_{n}=0$ ), and these results are not affected by the initial values. It should be noted that the Algorithm (5.2) without Tikhonov regularization terms is only weakly convergent.

(ii) It can be easily seen from Table 1 that the convergence speed of our proposed Algorithm (5.2) is faster than the ones without inertial terms proposed by Bot et al. [4].

Furthermore, we compare our Algorithm (5.2) with some previously known ones, see Table 2 for conditions and parameter values of those strong convergence algorithms. The parameters of Algorithm (5.2) are the same as above. Take $E_{n}<10^{-3}$ or maximum iteration 200 as a common stopping criterion. We implement this experiment by considering four different initial values $x_{0}, x_{1}$ (here, Case I: $x_{0}=\frac{t^{2}}{10}, x_{1}=\frac{\mathrm{e}^{t}}{2}$; Case II: $x_{0}=\frac{t^{2}}{10}, x_{1}=\frac{2^{t}}{2}+\frac{t^{2}}{10}$; Case III: $x_{0}=\frac{t^{2}}{10}, x_{1}=\frac{\mathrm{e}^{t}}{20}+\frac{t^{2}}{24}$; Case IV: $\left.x_{0}=\frac{t^{2}}{10}, x_{1}=\frac{2^{t}}{2}+\frac{\mathrm{e}^{t}}{20}\right)$. Computational results are reported in Table 3 and Figs. $1-4$.

TABLE 2. Conditions and parameter values of different strong convergence algorithms.

\begin{tabular}{|c|c|c|}
\hline Algorithms & Conditions & Parameter values \\
\hline $\begin{array}{c}\text { Bot et al. Alg. (2) [4] } \\
x_{n+1}=\beta_{n} x_{n}+\lambda_{n}\left(T\left(\beta_{n} x_{n}\right)-\beta_{n} x_{n}\right)\end{array}$ & $\begin{array}{l}\text { (i) } \beta_{n} \subset(0,1], \lambda_{n} \subset(0,1] \text {; } \\
\text { (ii) } \lim _{n \rightarrow+\infty} \beta_{n}=1, \sum_{n=0}^{\infty}\left(1-\beta_{n}\right)=+\infty \text {; } \\
\text { (iii) } \sum_{n=1}^{\infty}\left|\beta_{n}-\beta_{n-1}\right|<\infty \text {; } \\
\text { (iv) } \liminf _{n \rightarrow+\infty} \lambda_{n}>0 \text { and } \sum_{n=1}^{\infty}\left|\lambda_{n}-\lambda_{n-1}\right|<\infty \text {. }\end{array}$ & $\beta_{n}=\frac{n}{n+1}, \lambda_{n}=0.9$ \\
\hline $\begin{array}{r}\text { Yao and Cho Alg. (3.7) [7] } \\
x_{n+1}=\alpha_{n}\left(\lambda_{n} x_{n}\right)+\left(1-\alpha_{n}\right) T x_{n}\end{array}$ & $\begin{array}{l}\text { (i) } \alpha_{n} \subset(0,1), \lambda_{n} \subset(0,1) \text {; } \\
\text { (ii) } \lim _{n \rightarrow \infty} \alpha_{n}=0 \text { and } \lim _{n \rightarrow \infty} \lambda_{n}=1 \text {; } \\
\text { (iii) } \sum_{n=0}^{\infty}\left|\alpha_{n}-\alpha_{n-1}\right|<\infty, \sum_{n=0}^{\infty}\left|\lambda_{n}-\lambda_{n-1}\right|<\infty \text {; } \\
\text { (iv) } \sum_{n=0}^{\infty}\left(1-\lambda_{n}\right) \alpha_{n}=\infty\end{array}$ & $\alpha_{n}=\frac{1}{\sqrt{n+1}}, \lambda_{n}=1-\frac{1}{\sqrt{n+1}}$ \\
\hline $\begin{array}{l}\quad \text { Tan et al. Alg. (13) }[28] \\
\left\{\begin{array}{l}w_{n}=x_{n}+\theta_{n}\left(x_{n}-x_{n-1}\right) \\
y_{n}=\beta_{n} w_{n}+\left(1-\beta_{n}\right) T w_{n} \\
x_{n+1}=\gamma_{n} f\left(x_{n}\right)+\left(1-\gamma_{n}\right) y_{n}\end{array}\right.\end{array}$ & $\begin{array}{l}\text { (i) } \beta_{n} \subset(0,1), \gamma_{n} \subset(0,1) \text {; } \\
\text { (ii) } \lim _{n \rightarrow+\infty} \frac{\theta_{n}}{\gamma_{n}}\left\|x_{n}-x_{n-1}\right\|=0 \text {; } \\
\text { (iii) } \lim _{n \rightarrow \infty} \gamma_{n}=0, \sum_{n=0}^{\infty} \gamma_{n}=\infty\end{array}$ & $\begin{array}{l}\theta_{n} \text { update by (3.20) with } \xi_{n}=\frac{10}{(n+1)^{2}}, \eta=4, \\
\beta_{n}=\frac{1}{100(n+1)^{2}}, \gamma_{n}=\frac{1}{n+1}, f\left(x_{n}\right)=0.9 x_{n} .\end{array}$ \\
\hline $\begin{array}{c}\quad \text { Dong et al. Alg. (22) [11] } \\
\left\{\begin{array}{l}w_{n}=x_{n}+\alpha_{n}\left(x_{n}-x_{n-1}\right), \\
y_{n}=\left(1-\beta_{n}\right) w_{n}+\beta_{n} T w_{n}, \\
C_{n}=\left\{z \in \mathscr{H}:\left\|y_{n}-z\right\| \leq\left\|w_{n}-z\right\|\right\}, \\
Q_{n}=\left\{z \in \mathscr{H}:\left\langle x_{n}-z, x_{n}-x_{0}\right\rangle \leq 0\right\}, \\
x_{n+1}=P_{C_{n} \cap Q_{n}} x_{0} .\end{array}\right.\end{array}$ & $\begin{array}{l}\text { (i) } \alpha_{n} \subset\left[\alpha_{1}, \alpha_{2}\right], \alpha_{1} \in(-\infty, 0], \alpha_{2} \in[0, \infty) \text {; } \\
\text { (ii) } \beta_{n} \subset[\beta, 1], \beta \in(0,1] .\end{array}$ & $\alpha_{n}=0.5, \beta_{n}=0.9$ \\
\hline $\begin{array}{c}\text { Shehu et al. Alg. } 3.1[25] \\
\left\{\begin{array}{l}y_{n}=x_{n}+\theta_{n}\left(x_{n}-x_{n-1}\right) \\
x_{n+1}=\alpha_{n} x_{0}+\beta_{n} y_{n}+\gamma_{n} T y_{n}+e_{n}\end{array}\right.\end{array}$ & $\begin{array}{l}\text { (i) } \alpha_{n} \subset(0,1), \beta_{n} \subset(0,1), \gamma_{n} \subset(0,1) \text {; } \\
\text { (ii) } \lim _{n \rightarrow \infty} \alpha_{n}=0, \sum_{n=1}^{\infty} \alpha_{n}=\infty, \lim _{n \rightarrow \infty} \frac{\xi_{n}}{\alpha_{n}}=0 \text {; } \\
\text { (iii) } \alpha_{n}+\beta_{n}+\gamma_{n}=1 \text { and } \liminf _{n \rightarrow \infty} \beta_{n} \gamma_{n}>0 \text {; } \\
\text { (iv) Either } \sum_{n=1}^{\infty}\left\|e_{n}\right\|<\infty \text { or } \lim _{n \rightarrow \infty} \frac{\left\|e_{n}\right\|}{\alpha_{n}}=0\end{array}$ & $\begin{array}{l}\theta_{n} \text { update by (3.20) with } \xi_{n}=\frac{10}{(n+1)^{2}}, \eta=4, \\
\alpha_{n}=\frac{1}{n+1}, \beta_{n}=\gamma_{n}=\frac{n}{2(n+1)}, e_{n}=0 .\end{array}$ \\
\hline
\end{tabular}


TABLE 3. Numerical results of different algorithms for Example 5.1.

\begin{tabular}{|c|c|c|c|c|c|c|c|c|c|c|c|c|}
\hline \multirow[b]{2}{*}{ Cases } & \multicolumn{2}{|c|}{ Our Alg. (5.2) } & \multicolumn{2}{|c|}{ Bot et al. Alg. (2) } & \multicolumn{2}{|c|}{ Yao and Cho Alg. (3.7) } & \multicolumn{2}{|c|}{ Tan et al. Alg. (13) } & \multicolumn{2}{|c|}{ Dong et al. Alg. (22) } & \multicolumn{2}{|c|}{ Shehu et al. Alg. 3.1} \\
\hline & Iter. & Time(s) & Iter. & Time(s) & Iter. & Time(s) & Iter. & Time(s) & Iter. & Time(s) & Iter. & Time(s) \\
\hline I & 10 & 3.7068 & 11 & 3.4814 & 13 & 3.8794 & 26 & 10.0685 & $>200$ & 86.9974 & $>200$ & 73.6488 \\
\hline II & 6 & 2.2128 & 9 & 2.7769 & 10 & 2.9613 & 17 & 6.5503 & $>200$ & 87.6840 & $>200$ & 73.2906 \\
\hline III & 5 & 1.9614 & 7 & 2.1968 & 7 & 2.2379 & 14 & 5.5790 & $>200$ & 92.2960 & $>200$ & 74.4269 \\
\hline IV & 7 & 2.6032 & 9 & 2.7078 & 10 & 2.9121 & 19 & 7.8907 & $>200$ & 91.3503 & $>200$ & 74.3966 \\
\hline
\end{tabular}

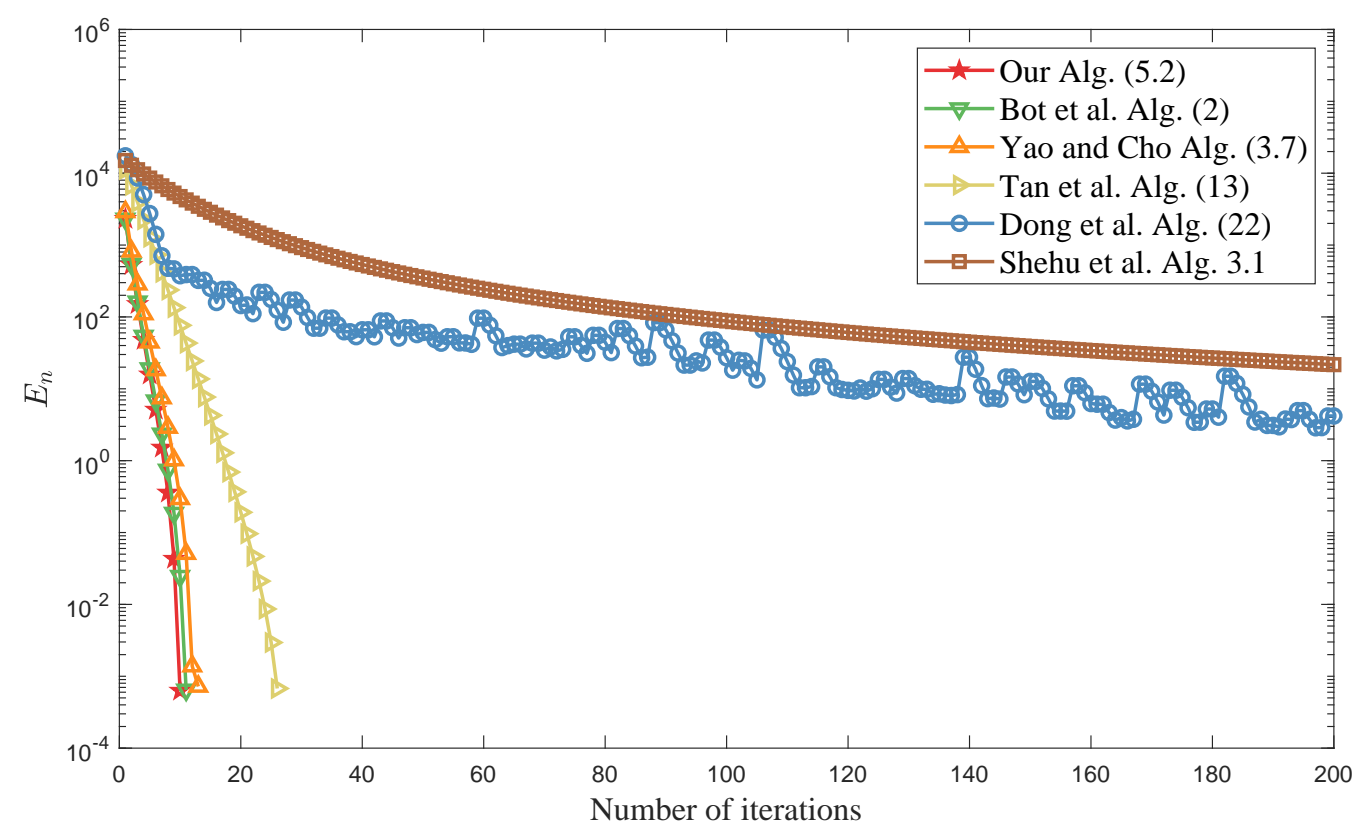

FIGURE 1. Numerical behavior of $\left\{E_{n}\right\}$ for Example 5.1 in (Case I).

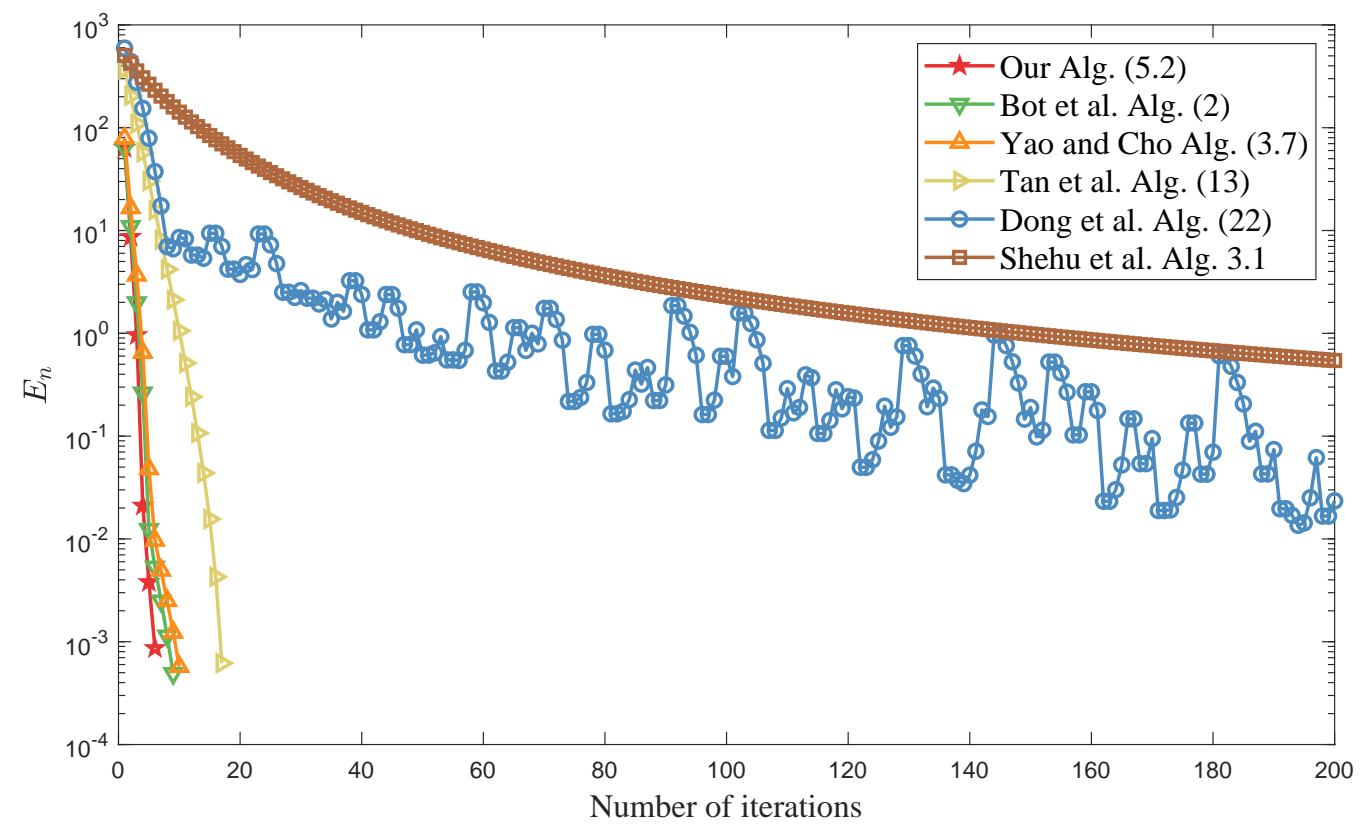

FiguRE 2. Numerical behavior of $\left\{E_{n}\right\}$ for Example 5.1 in (Case II). 


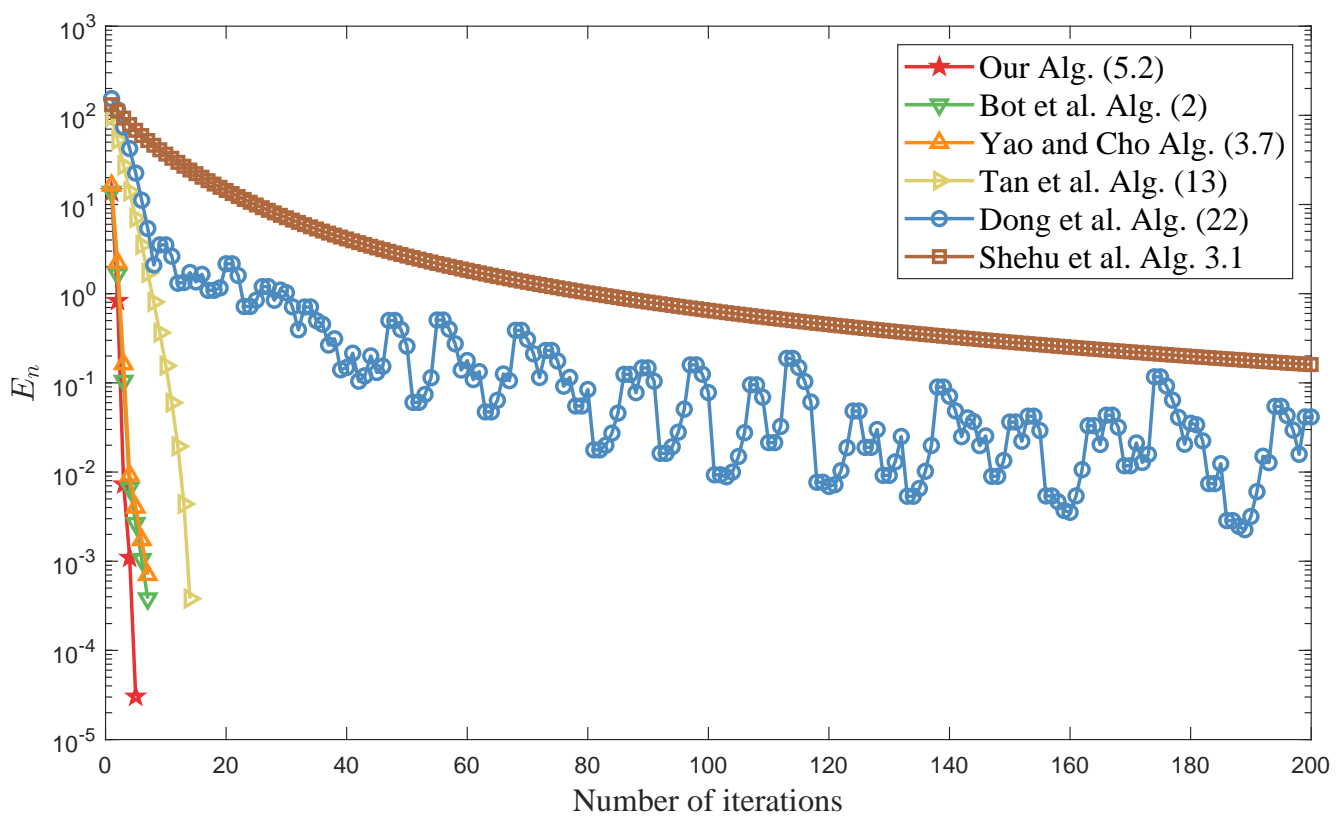

FIGURE 3. Numerical behavior of $\left\{E_{n}\right\}$ for Example 5.1 in (Case III).

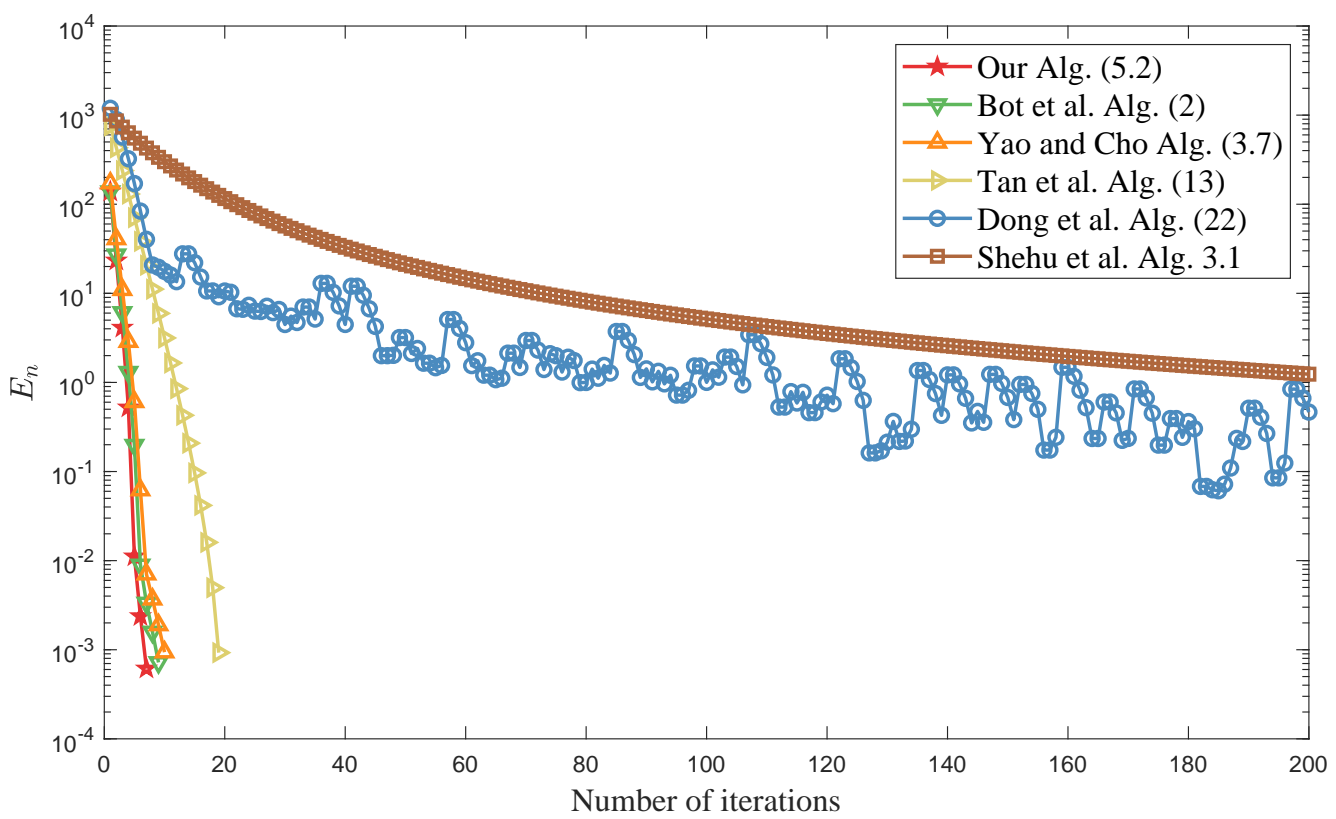

FIGURE 4. Numerical behavior of $\left\{E_{n}\right\}$ for Example 5.1 in (Case IV).

Remark 5.2. (i) It is observed from Table 3 and Figs. 1-4 that our Algorithm (5.2) involving Tikhonov regularization terms is convergent, easy to implement, and very fast.

(ii) Our proposed Algorithm (5.2) improves some previously known algorithms and results from the perspective of the number of iterations and CPU time.

(iii) Since the choice of initial values does not affect the number of iterations and CPU time required to obtain the desired results, our proposed algorithm is reliable and robust. 
Example 5.2. In next example, we consider the variational inequality problem described in (4.4). We set $A:=\left(a_{i j}\right)_{1 \leq i, j \leq m}$ to be a square matrix of $m \times m$, and its elements are given by:

$$
a_{i j}= \begin{cases}1, & \text { if } j=m+1-i \text { and } j<i \\ -1, & \text { if } j=m+1-i \text { and } j>i \\ 0, & \text { otherwise }\end{cases}
$$

Take the set $C:=\left\{-5 \leq x_{i} \leq 5, i=1,2, \ldots, m\right\}$ as a feasible region. We know that $x^{*}=(0, \ldots, 0)$ is a solution of this problem. This example has been studied by many authors. In order to compare the efficiency between different algorithms, we consider our proposed Algorithm (4.5), the extragradient method (EGM) in [14], the subgradient extragradient method (SEGM) in [8], and the new inertial subgradient extragradient method (NISEGM) in [12]. In Algorithm EGM and Algorithm SEGM, we take $\lambda=0.7$. In Algorithm NISEGM, we set $\alpha_{n}=0.1, \tau_{n}=\frac{n}{(n+1)^{1.1}}$ and $\lambda_{n}=0.8$. In our Algorithm (4.5), let inertial parameter $\theta_{n}$ be updated by (3.20) with $\xi_{n}=\frac{10}{(n+1)^{2}}$ and $\eta=21, \beta_{n}=0.9, \lambda=0.7$ and Tikhonov regularization parameters $\alpha_{n}=\frac{n}{(n+1)^{1.1}}$. We take different values of dimension $m$ into consideration and $E_{n}=\left\|x_{n}-x^{*}\right\|_{2}<10^{-4}$ as the stopping criterion. The initial values $x_{0}, x_{1}$ are randomly generated in MATLAB. Table 4 shows the numerical performance of these algorithms in different dimensions. The evolution trend of their iteration errors $E_{n}$ with the number of iterations is plotted in Fig. 5.

TABLE 4. Comparison between Algorithm (4.5), NISEGM, EGM and SEGM for Example 5.2.

\begin{tabular}{|c|c|c|c|c|c|c|c|c|}
\hline \multirow[b]{2}{*}{$m$} & \multicolumn{2}{|c|}{ Algorithm (4.5) } & \multicolumn{2}{|c|}{ NISEGM } & \multicolumn{2}{|c|}{ EGM } & \multicolumn{2}{|c|}{ SEGM } \\
\hline & Iter. & Time(s) & Iter. & Time(s) & Iter. & Time(s) & Iter. & Time(s) \\
\hline 100 & 11 & & 85 & 0.0148 & 91 & & 94 & \\
\hline 1000 & 12 & 0.0234 & 92 & 0.1234 & 99 & 0.1719 & 101 & 0.2016 \\
\hline 2000 & 14 & 0.0588 & 94 & 0.6591 & 101 & 0.7023 & 104 & 0.7473 \\
\hline 5000 & 15 & 0.3262 & 97 & 3.9382 & 105 & 4.3697 & 107 & 4.4306 \\
\hline
\end{tabular}

Remark 5.3. (i) From Table 4 and Fig. 5, we know that our Algorithm (4.5) is convergent, easy to implement, and most important very fast. Moreover, the choice of dimensions does not affect our computational results, one can notice that the jumping change of the dimension has little effect on the number of iterations required by our algorithm.

(ii) In both of the CPU time and the number of iterations, we also find that our proposed Algorithm (4.5) outperforms some previously known algorithms and results.

Example 5.3. In this example, we consider using the Algorithm (4.3) to solve the linear inverse problem that occurs in signal processing, which leads us to study the following problem $\mathbf{A} \mathbf{x}_{0}=$ $\mathbf{b}+\mathbf{w}$, where $\mathbf{A} \in \mathbb{R}^{M \times N}$ represents a known linear operator, $\mathbf{b} \in \mathbb{R}^{M}$ is a known blurred signal, $\mathbf{w} \in \mathbb{R}^{M}$ is the assumed known noise vector, and $\mathbf{x}_{0} \in \mathbb{R}^{N}$ with $k(k \ll N)$ non-zero elements is the "true" and unknown sparse signal to be recovered. In this problem, we consider $\mathbf{A}$ as a filter, and $\mathbf{A x}=\varphi \star \mathbf{x}$, where $\varphi$ is a second derivative of Gaussian. In addition, we assume $M=N$. In order to obtain an approximate solution of signal $\mathbf{x}_{0}$, we use the following $\ell_{1}$-norm regularized least squares model

$$
\min _{\mathbf{x} \in \mathbb{R}^{N}} \Phi(\mathbf{x})=\frac{1}{2}\|\mathbf{b}-\mathbf{A x}\|^{2}+\lambda\|\mathbf{x}\|_{1},
$$




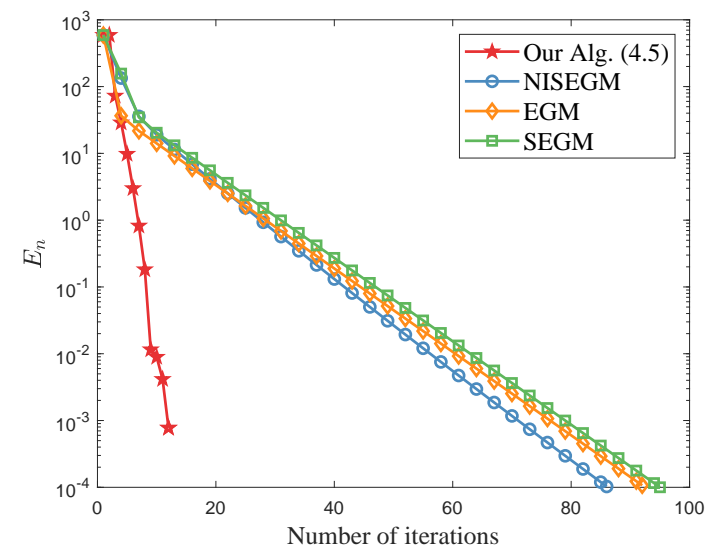

(a) $m=100$

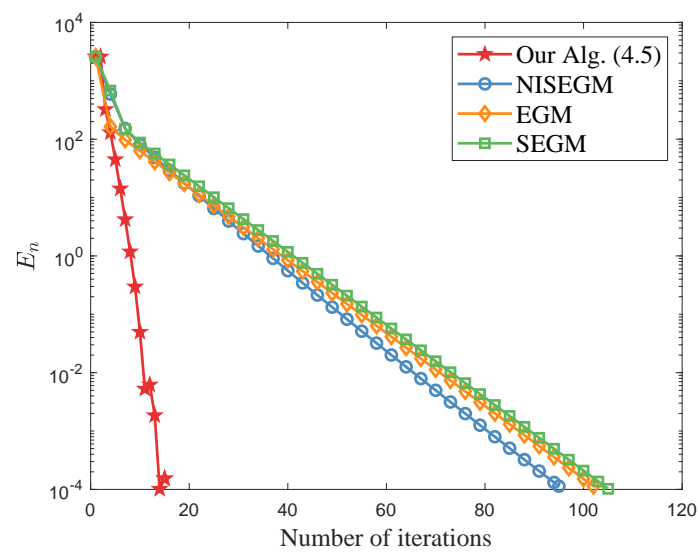

(c) $m=2000$

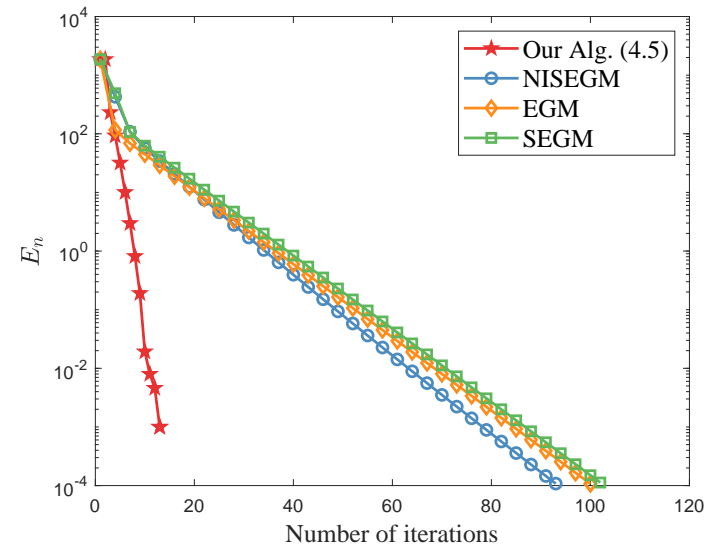

(b) $m=1000$

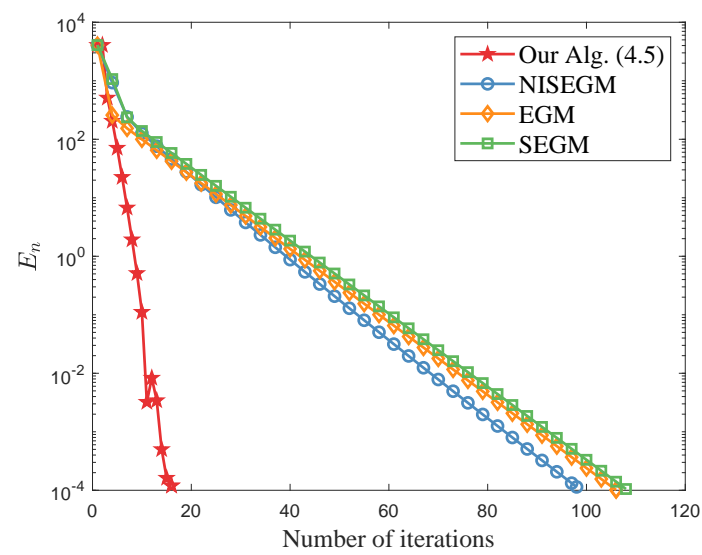

(d) $m=5000$

FIGURE 5. Numerical behavior of $\left\{E_{n}\right\}$ with different dimensions for Example 5.2.

where $\lambda$ is a positive regularization parameter that controls the trade-off between sparsity and reconstruction fidelity. Model (5.3) is referred to as the basis pursuit denoising, which has wide applications in image processing, compressed sensing, statistics, and machine learning. Many authors constructed efficient computational algorithms to solve it. One can notice that (5.3) is a special case of minimizing $\{f+g\}$, where $f(\mathbf{x})=\lambda\|\mathbf{x}\|_{1}$ and $g(\mathbf{x})=\frac{1}{2}\|\mathbf{b}-\mathbf{A x}\|^{2}$. The proximal operator of $f$ has a simple solution $\operatorname{prox}_{\gamma f}\left(\mathbf{x}_{k}\right)=\max \left(0,1-\lambda \gamma /\left|\mathbf{x}_{k}\right|\right) \mathbf{x}_{k}$, which is so-called "soft thresholding". Furthermore, it is easy to check that $g$ is a smooth function with $\left\|\mathbf{A}^{*} \mathbf{A}\right\|$-Lipschitz continuous gradient $\nabla g(\mathbf{x})=\mathbf{A}^{*}(\mathbf{A x}-\mathbf{b})$.

We compare our Algorithm (4.3) with another strong convergence algorithm proposed by Gibali and Thong in [13] and the forward-backward algorithm (FBA) in [9]. In all algorithms, set $N=1000, k=30, \mathbf{w}$ is a Gaussian white noise with variance $10^{-2}$, and regularization parameter $\lambda=\frac{1}{2}$ in (5.3). In Algorithm FBA, set step size $\gamma=1.9 / L$. In Gibali and Thong's Algorithm 1, take step size $\gamma=1.9 / L, \alpha_{n}=\frac{1}{n+1}, \beta_{n}=\frac{n}{2(n+1)}$ and $\mu=0.5$. In our Algorithm (4.3), let $\theta_{n}$ be updated by (3.20) with $\xi_{n}=\frac{10}{(n+1)^{2}}$ and $\eta=4, \beta_{n}=0.7, \gamma=1.9 / L$ and Tikhonov regularization parameters $\alpha_{n}=\frac{1}{100(n+1)}$. We take maximum iteration $5 \times 10^{4}$ as a common stopping criterion. Results of these calculations are given in Fig. 6. 


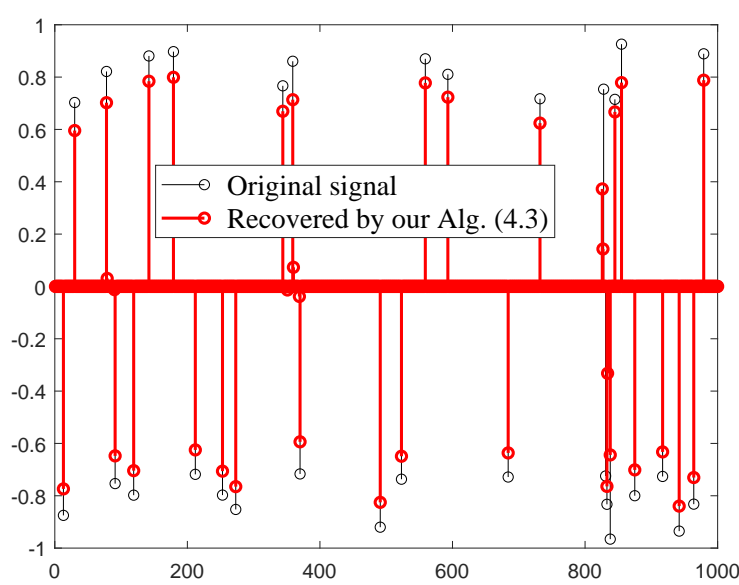

(a) Recovery results by our Alg. (4.3)

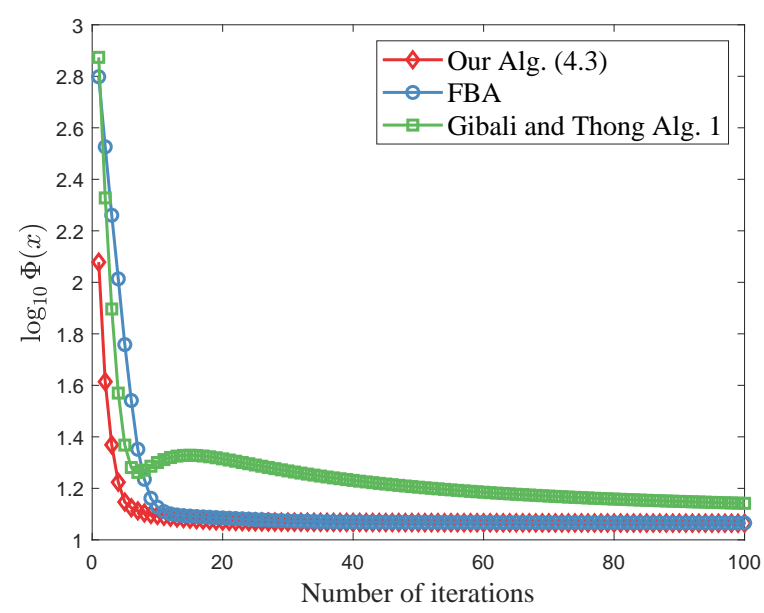

(b) Iterative process of $\Phi(x)$

FIGURE 6. Numerical results for Example 5.3.

Remark 5.4. (i) Fig. 6 shows that the suggested Algorithm (4.3) outperforms the Algorithm 1 proposed by Gibali and Thong [13] and the forward-backward algorithm [9] in terms of computational performance and accuracy.

(ii) It is worth noting that the forward-backward algorithm is weakly convergent in infinitedimensional Hilbert spaces. However, our proposed Algorithm (4.3) is strongly convergent in infinite-dimensional Hilbert spaces.

\section{FinAL REMARKS}

In this paper, we proposed a strong convergent inertial Mann-like algorithm for nonexpansive mappings in infinite-dimensional Hilbert spaces and obtained strong convergence theorems under some suitable conditions. In addition, we derived two strong convergence algorithms of monotone inclusion problems and variational inequality problems. Applications to split feasibility problems, variational inequality problems and signal recovery were considered. Numerical experiments confirmed that the theoretical results of our proposed algorithms.

\section{REFERENCES}

[1] N.T. An, N.M. Nam, X. Qin, Solving $k$-center problems involving sets based on optimization techniques, J. Global Optim. 76 (2020), 189-209.

[2] Q.H. Ansari, M. Islam, J.C. Yao, Nonsmooth variational inequalities on Hadamard manifolds, Appl. Anal. 99 (2020), 340-358.

[3] H. Attouch, Viscosity solutions of minimization problems, SIAM J. Optim. 6 (1996), 769-806.

[4] R.I. Boț, E.R. Csetnek, D. Meier, Inducing strong convergence into the asymptotic behaviour of proximal splitting algorithms in Hilbert spaces, Optim. Methods Softw. 34 (2019), 489-514.

[5] A. Beck, M. Teboulle, A fast iterative shrinkage-thresholding algorithm for linear inverse problems, SIAM J. Imaging Sci. 2 (2009), 183-202.

[6] H.H. Bauschke, P.L. Combettes, Convex Analysis and Monotone Operator Theory in Hilbert Spaces, Springer, Berlin, 2011.

[7] S.S. Chang, C.F. Wen, J.C. Yao, Common zero point for a finite family of inclusion problems of accretive mappings in Banach spaces, Optimization 67 (2018), 1183-1196. 
[8] Y. Censor, A. Gibali, S. Reich, The subgradient extragradient method for solving variational inequalities in Hilbert space, J. Optim. Theory Appl. 148 (2011), 318-335.

[9] P.L. Combettes, V.R. Wajs, Signal recovery by proximal forward-backward splitting, Multiscale Model. Simul. 4 (2005), 1168-1200.

[10] Q.L. Dong, Y.Y. Lu, J. Yang, The extragradient algorithm with inertial effects for solving the variational inequality, Optimization 65 (2016), 2217-2226.

[11] Q.L. Dong, H.B. Yuan, Y.J. Cho, T.M. Rassias, Modified inertial Mann algorithm and inertial CQ-algorithm for nonexpansive mappings, Optim. Lett. 12 (2018), 87-102.

[12] J. Fan, L. Liu, X. Qin, A subgradient extragradient algorithm with inertial effects for solving strongly pseudomonotone variational inequalities, Optimization 69 (2020), 2199-2215.

[13] A. Gibali, D.V. Thong, Tseng type methods for solving inclusion problems and its applications, Calcolo 55 (2018), 49.

[14] G.M. Korpelevich, The extragradient method for finding saddle points and other problems, Ekonomikai Matematicheskie Metody. 12 (1976), 747-756.

[15] L. Liu, A hybrid steepest descent method for solving split feasibility problems involving nonexpansive mappings, J. Nonlinear Convex Anal. 20 (2019), 471-488.

[16] N. Lehdili, A. Moudafi, Combining the proximal algorithm and Tikhonov regularization, Optimization 37 (1996), 239-252.

[17] P.E. Maingé, Convergence theorems for inertial KM-type algorithms, J. Comput. Appl. Math. 219 (2008), 223-236.

[18] B.T. Polyak, Some methods of speeding up the convergence of iteration methods, USSR Comput. Math. Math. Phys. 4 (1964), 1-17.

[19] X. Qin, N.T. An, Smoothing algorithms for computing the projection onto a Minkowski sum of convex sets, Comput. Optim. Appl. 74 (2019), 821-850.

[20] X. Qin, A. Petrusel, J.C. Yao, CQ iterative algorithms for fixed points of nonexpansive mappings and split feasibility problems in Hilbert spaces, J. Nonlinear Convex Anal. 19 (2018), 157-165.

[21] X. Qin, L. Wang, J.C. Yao, Inertial splitting method for maximal monotone mappings, J. Nonlinear Convex Anal. 21 (2020), 2325-2333.

[22] D.R. Sahu, J.C. Yao, The prox-Tikhonov regularization method for the proximal point algorithm in Banach spaces, J. Global Optim. 51 (2011), 641-655.

[23] D.R. Sahu, Q.H. Ansari, J.C. Yao, The prox-Tikhonov-like forward-backward method and applications, Taiwanese J. Math. 19 (2015), 481-503.

[24] Y. Shehu, X.H. Li, Q.L. Dong, An efficient projection-type method for monotone variational inequalities in Hilbert spaces, Numer. Algorithm 84 (2020), 365-388.

[25] Y. Shehu, Q.S. Iyiola, F.U. Ogbuisi, Iterative method with inertial terms for nonexpansive mappings: applications to compressed sensing, Numer Algorithm 83 (2020), 1321-1347.

[26] B. Tan, S. Xu, S. Li, Inertial shrinking projection algorithms for solving hierarchical variational inequality problems, J. Nonlinear Convex Anal. 21 (2020), 871-884.

[27] B. Tan, S. Xu, S. Li, Inertial hybrid and shrinking projection algorithms for solving variational inequality problems, J. Nonlinear Convex Anal. 21 (2020), 2193-2206.

[28] B. Tan, Z. Zhou, S. Li, Strong convergence of modified inertial Mann algorithms for nonexpansive mappings, Mathematics 8 (2020), 462.

[29] H.K. Xu, Iterative algorithms for nonlinear operators, J. Lond. Math. Soc. 66 (2002), 240-256. 